Reprod. Nutr. Dévelop., 1983, 23 (2 B), 403-411.

\title{
Comparaison du mécanisme d'action de l'insuline et de la prolactine
}

\author{
L.-M. HOUBEDINE, J. DJIANE, B. TEYSSOT, P. A. KELLY $\left({ }^{*}\right)$
}

Laboratoire de Physiologie de la Lactation, I.N.R.A., 78350 Jouy-en-Josas, France.

(*) Département d'Endocrinologie moléculaire, Centre Hospitalier de l'Université Laval, Québec, Canada.

Summary. A comparison of the action mechanism of insulin and prolactin.

One of the major actions of prolactin in the mammary cell is to activate the expression of casein genes by enhancing the transcription rate of the genes. Anti-prolactin receptor antibodies can mimic prolactin action when added to mammary cells, suggesting that a relay is formed at the membrane level and transferred to the target genes. None of the classical hormone intracellular relays can account for the transfer of the prolactin message. The incubation of membranes from various tissues containing prolactin receptor with prolactin provokes the release of a factor which specifically accelerates the transcription of the $\beta$-casein gene in isolated mammary nuclei. The factor is thermostable, inactivated by trypsin and is eluted from G-10 Sephadex as a molecule smaller than 1000 daltons. The action of the factor is prevented by phosphatase inhibitors. These results are strikingly reminiscent of those obtained with insulin which activates enzymes via a factor released from the membranes and which dephosphorylates the enzymes. The analogy between the action mechanism of prolactin and insulin is discussed.

\section{Introduction.}

II n'est pas habituel de comparer les mécanismes d'action de l'insuline et de la prolactine. Jusqu'à ces derniers temps, ces deux hormones ne semblaient guère avoir en commun que le fait d'être deux hormones protéiques possédant des récepteurs périphériques dans leurs cellules cibles et n'ayant pour relais intracellulaire aucune des molécules classiquement envisagées pour les autres hormones (AMP cyclique, $\mathrm{Ca}^{++}$, prostaglandines...). Des expériences récentes ont fait apparaître des analogies beaucoup plus frappantes. Ces deux hormones ont en effet la capacité de provoquer la libération, à partir des membranes, d'une molécule qui mime leur action dans des systèmes subcellulaires. Dans ces deux cas, ces molécules ont des critères de spécificité d'action qui les placent en bonne position pour être les relais intracellulaires respectifs de ces deux hormones. Ce rapport se propose de se livrer à un examen comparatif et critique des données encore fragmentaires concernant ces deux relais hormonaux. Les questions fon- 
damentales qui paraissent actuellement se poser sont les suivantes : les deux hormones procèdent-elles d'un même mécanisme pour la formation de leur relais respectif à partir des membranes? Les deux relais sont-ils une seule et même molécule ou présentent-ils seulement quelques analogies? Les deux relais agissent-ils par l'intermédiaire de mécanismes communs ? En d'autres termes, les observations faites avec l'insuline et la prolactine sont-elles susceptibles d'être généralisées?

\section{Transfert de l'information de la prolactine ou de l'insuline à l'intérieur de leurs cellules cibles.}

a) Effet hormono-mimétique des anticorps anti-récepteurs. -- Nous avons montré récemment (Djiane et al., 1981) que des anticorps dirigés contre le récepteur de la prolactine étaient doués de propriétés prolactino-mimétiques sur la cellule mammaire. En effet, leur addition à faible concentration $(0,1$ à $1 \%)$ au milieu de culture d'explants mammaires prélevés au cours de la pseudogestation chez la lapine provoque l'activation de la biosynthèse des caséines ainsi que l'accumulation de leurs ARN messagers. Ces expériences suggèrent que la prolactine n'est plus nécessaire après son interaction avec son récepteur pour que l'effet hormonal s'exprime. En d'autres termes, il apparaît que le récepteur a pour rôle essentiel d'assurer le transfert de la stimulation hormonale plutôt que d'assurer un simple transport de l'hormone vers des sites intracellulaires effecteurs, comme cela pouvait être suggéré après la mise en évidence de l'endocytose des complexes prolactine-récepteur (Nolin et Bogdanove, 1980). Le mécanisme le plus probable pour le transfert de l'information hormonale suppose donc qu'il y a génération au niveau membranaire d'un relais de l'hormone ou " second messager $\gg$.

Des travaux récents (Kahn et al., 1977 ; Baldwin et al., 1980) ont clairement démontré que des anticorps anti-récepteurs de l'insuline sont doués de propriétés insulino-mimétiques. Ces propriétés sont liées à la bivalence des immunoglobulines. Ce fait a conduit à formuler l'hypothèse que le mécanisme de transfert de la stimulation insulinique pourrait faire intervenir une agrégation des récepteurs entre eux (Kahn et al., 1978). La conclusion essentielle qui peut être tirée des expériences utilisant les anti-récepteurs de la prolactine et de l'insuline est qu'il doit exister un relais d'origine membranaire pour rendre compte de l'action cellulaire de ces deux hormones.

b) Les relais possibles pour la prolactine ou l'insuline. - De nombreux travaux ont établi qu'aucun des relais membranaires classiques ne peut rendre compte des effets soit de l'insuline, soit de la prolactine sur leurs cellules cibles. Ont été ainsi examinés le cAMP, le cGMP, les ions $\mathrm{Ca}^{++}$ou $\mathrm{K}^{+}$, les prostaglandines (Houdebine, 1980).

Les travaux de Larner et al. (1979), puis ceux de Seals et Jarett (1980) sur le mécanisme d'action de l'insuline, semblent avoir ouvert une autre voie. En effet ces auteurs ont démontré que l'insuline était capable de provoquer l'émission d'un relais soluble de nature probablement peptidique responsable de l'activation via une déphosphorylation de certaines enzymes intracellulaires caractéristique de 
l'effet insulinique. C'est ainsi que la pyruvate déshydrogénase au niveau mitochondrial ou la glycogène synthétase se trouve activée, ce qui ferait jouer au relais intracellulaire potentiel de l'insuline un rôle assez général dans les processus de déphosphorylation de certaines protéines enzymatiques, rôle symétrique de celui de I'AMP cyclique. Un tel rôle ne s'oppose pas au fait que l'insuline peut au contraire favoriser la phosphorylation d'autres protéines, étant donnée la multiplicité des mécanismes contrôlés par ces phosphorylations (Cohen, 1982).

En ce qui concerne la prolactine, la démonstration de l'émission d'un relais membranaire impliqué dans son mécanisme d'action intracellulaire a pu être obtenue grâce à l'utilisation de noyaux mammaires isolés ayant conservé une bonne partie de leurs capacités fonctionnelles (Teyssot et Houdebine, 1980-1981). Il a été montré que des noyaux extraits de glandes mammaires d'animaux traités par la prolactine étaient capables de synthétiser in vitro des ARN messagers des caséines et ceci d'une façon plus importante que des noyaux provenant d'animaux n'ayant pas subi de traitement. Dans ces expériences, les ARN messagers ont été néosynthétisés à partir de nucléotides parmi lesquels le CTP était mercuré, permettant ainsi d'isoler ces ARN messagers néosynthétisés sur une colonne de SH Sépharose.

Nous avons montré récemment (Teyssot et al., 1981) qu'il était possible d'activer le phénomène de transcription directement in vitro par l'adjonction à la préparation de noyaux, de surnageants de membranes cellulaires préalablement incubées avec de la prolactine. Dans ces conditions, toutes les hormones connues pour leur activité lactogène chez la lapine (oPRL, hGH, oPL) se sont révélées capables d'induire la libération à partir des membranes possédant le récepteur de la prolactine, d'un facteur soluble capable de relancer in vitro la transcription des gènes des caséines précédemment déprimée in vivo par un traitement hypoprolactinémiant (bromocriptine). Les hormones lactogènes ajoutées seules, directement, aux noyaux ne produisent aucun effet (Teyssot et al., 1981).

Les caractéristiques physicochimiques de ce relais potentiel de la prolactine ont été examinées. La figure 1 illustre la détermination approximative de son poids moléculaire par filtration sur Séphadex G 10. Des membranes microsomiales préparées à partir de glandes mammaires de lapines en lactation ont été incubées avec de la prolactine, puis le surnageant de cette incubation a été concentré et déposé sur une colonne Séphadex G 10. Le volume d'élution a été recueilli en 6 fractions dont les capacités d'activation de la transcription des gènes des caséines, sur des préparations de noyaux mammaires isolés, ont été mesurées. Les fractions 3 et 4 correspondant à des molécules d'un poids moléculaire faible $(<1000 \mathrm{~d})$ sont les plus actives, puisque la transcription du gène de la caséine- $\beta$ de lapine se trouve jusqu'à 7 fois plus active que dans des préparations de noyaux incubés avec la fraction 1 ou 2. Cet effet sur la transcription des gènes des caséines avait été établi comme l'un des plus spécifiques de l'effet prolactine sur la cellule mammaire et l'on peut considérer que le relais intracellulaire de cette hormone, pour cette action, est présent dans les fractions 3 et 4 alors que les fractions 1 et 2 qui contiennent la prolactine sont totalement inactives.

La spécificité de ce phénomène a été soigneusement analysée. Seules les hormones lactogènes sont capables d'induire la libération d'un tel relais à partir 


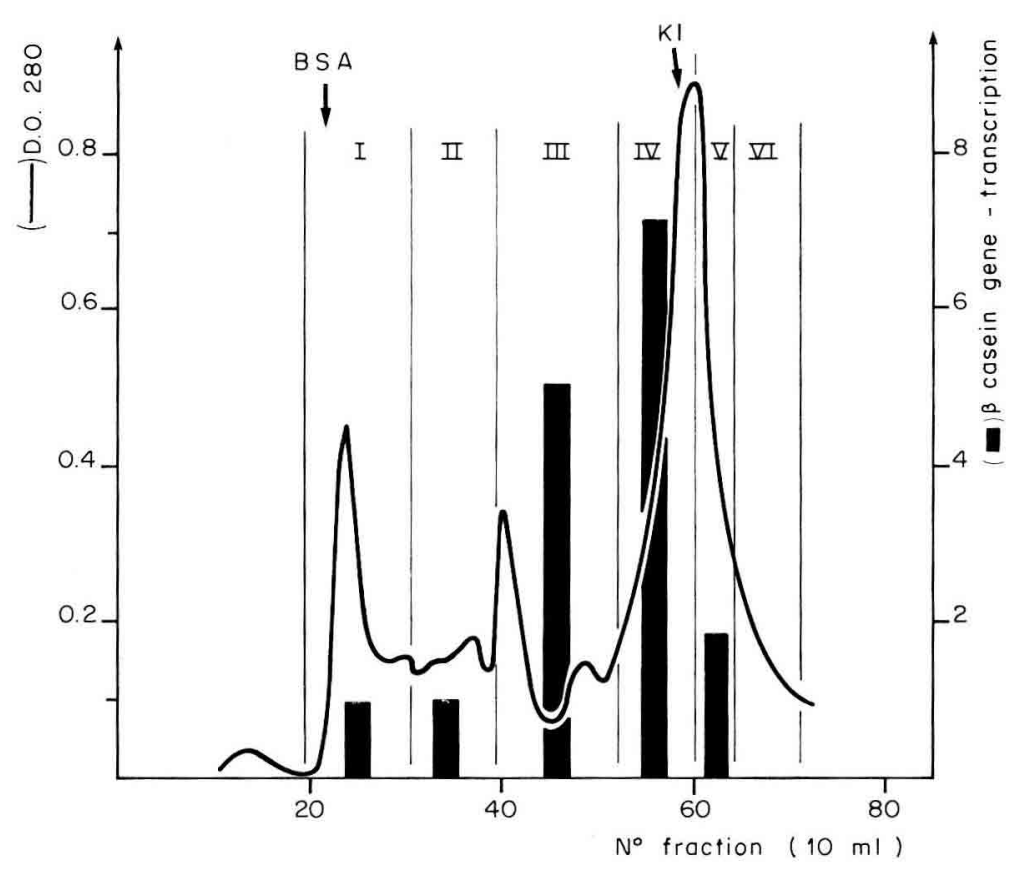

FIG. 1. - Fractionnement du surnageant de membranes mammaires incubées avec la prolactine. Le surnageant de membranes, après lyophilisation, a été déposé sur une colonne de Séphadex G$10(\mathrm{~h}=60 \mathrm{~cm} ; \emptyset=3,5 \mathrm{~cm})$, et l'élution a été réalisée dans l'eau. L'éluat a été divisé en 6 fractions qui ont été lyophilisées puis ajoutées à des noyaux mammaires isolés et incubés en présence de $\mathrm{Hg}$ CTP. Les RNA mercurés ont alors été sélectivement retenus puis élués sur une colonne de thiopropyl Sépharose. Les séquences mRNA de la caséine- $\beta$ contenues dans l'éluat de cette colonne et reflétant le taux de transcription du gène correspondant dans les noyaux ont été évaluées à l'aide d'une sonde cDNA. Les valeurs sont rapportées à un standard obtenu avec les noyaux seuls.

des seules membranes qui contiennent le récepteur de la prolactine (glande mammaire, foie, ovaire, surrénales). Ce relais est probablement préexistant dans la membrane puisque l'interaction de la prolactine avec son récepteur est capable de le libérer sans nécessiter un substrat extérieur à la membrane. Toutefois, il acquiert une polarité hydrophile très marquée puisqu'il est aisément retrouvé dans ie surnageant des membranes. La nature protéique de ce relais est suggérée par sa sensibilité à la dégradation trypsique et sa thermostabilité par sa résistance à un traitement de $10 \mathrm{~min}$ à $100^{\circ} \mathrm{C}$.

Toutes les caractéristiques physicochimiques précédemment énoncées pour ce relais potentiel de la prolactine sont identiques à celles décrites pour le relais potentiel de l'insuline (Kiechle et al., 1981). Il paraît donc important de rechercher s'il n'y aurait pas identité des deux relais. Nous avons donc, dans cette optique, entrepris une étude visant à comparer l'effet de la prolactine et de I'insuline sur des membranes cellulaires contenant les deux types de récepteurs, ou un seul, et à analyser l'émission de relais actifs sur la transcription des gènes des caséines des noyaux de glande mammaire. Les résultats encore préliminaires 
suggèrent que l'insuline a un effet inconstant dans notre système et de toute façon beaucoup plus faible que celui de la prolactine. II reste toutefois que dans certains cas, en particulier en présence de membranes hépatiques qui contiennent les deux types de récepteurs (insuline et prolactine), l'insuline a été capable de provoquer l'émission d'un relais stimulant la transcription des gènes des caséines dans des noyaux mammaires et ceci avec une efficacité d'environ $50 \%$ de celle de la prolactine.

\section{Le mode d'action du relais de la prolactine.}

$\mathrm{S}^{\prime}$ il est maintenant généralement admis que la plupart des inducteurs, hormonaux ou non, activent leurs gènes cibles au moins en partie en augmentant le taux de transcription de ces gènes, dans aucun cas la chaîne complète des événements qui président à cette activation au niveau moléculaire n'a été décryptée. $S^{\prime}$ il est vraisemblable que des séquences nucléotidiques précédant la partie codante des gènes sur le DNA sont impliquées dans la reconnaissance des signaux hormonaux (Mulvihill et al., 1982 ; Fasel et al., 1982), l'intervention des changements conformationnels de la chromatine et des protéines chromatiniennes elles-mêmes est plus que probable (Mathis et al., 1980). La petite taille du relais intracellulaire de la prolactine rend encore plus improbable son interaction simple avec le DNA comme seul mécanisme pour l'activation des gènes des caséines.

Il est généralement admis que l'insuline active un certain nombre d'enzymes dans ses cellules cibles en les déphosphorylant, via l'activation d'une phosphatase (Cohen, 1982). II semble par ailleurs que le relais de l'insuline agisse selon ce même mode d'action dans un système subcellulaire (Cheng et al., 1980 ; Kiechle et al., 1981). Cette observation a grandement contribué à donner de la crédibilité à l'identité du relais de l'insuline. Etant donné les analogies qui semblent exister entre les relais de l'insuline et de la prolactine, il est permis de se demander si le relais de la prolactine n'agirait pas aussi en déphosphorylant des protéines. A peu près rien n'est connu sur le rôle de l'équilibre phosphorylationdéphosphorylation dans le mécanisme d'action de la prolactine. L'addition de dichloro-acétate de sodium à des milieux de culture de cellules mammaires isolées mime l'action de l'hormone (tabl. 1). Ce composé étant un inhibiteur des

TABLEAU 1

Action du dichloracétate de sodium sur l'accumulation des RNA messagers de la caséine- $\beta$ dans des cellules mammaires en culture. Dans tous les cas, les milieux de culture contenaient de l'insuline $(5 \mu \mathrm{g} / \mathrm{ml})$. La prolactine ovine et le dichloracétate de sodium étaient présents à la concentration de $100 \mathrm{ng} / \mathrm{ml}$ et $0,1 \mathrm{mM}$ respectivement. La quantité de RNA messagers de la caséine- $\beta$ a été déterminée à l'aide d'une sonde DNA complémentaire (Teyssot et al., (1981).

\begin{tabular}{cccc}
\hline Milieu de culture & Contrôle & Prolactine & $\begin{array}{c}\text { Dichloracétate } \\
\text { de sodium }\end{array}$ \\
\hline $\begin{array}{l}\text { Nombre de RNA messagers de la } \\
\text { caséine- } \beta \text { par cellule }\end{array}$ & 10 & 32 & 32 \\
\hline
\end{tabular}


Action de divers inhibiteurs de phosphatase sur la stimulation de la transcription du gène de la caséine- $\beta$ par le relais de la prolactine.

La technique est celle décrite dans la légende de la figure 1.

\begin{tabular}{|c|c|}
\hline Milieu d'incubation des noyaux & Taux de transcription \\
\hline Contrôle & 1 \\
\hline + Relais & 6 \\
\hline+ Relais + NaF $(75 \mathrm{mM})$ & 1 \\
\hline+ Relais + $\mathrm{NaCl}(75 \mathrm{mM})$ & 6 \\
\hline + Relais + Phosphosérine (50 mM) & 1 \\
\hline + Relais + p-nitrophénylphosphate $(5 \mathrm{mM})$ & 1,5 \\
\hline
\end{tabular}

protéines kinases (Whitehouse et al., 1974), on peut penser que le relais de la prolactine agit en inactivant une kinase mammaire. L'inhibiteur des kinases n'est toutefois que relativement peu actif lorsqu'il est ajouté directement sur les noyaux mammaires isolés. Par contre, divers inhibiteurs classiques des phosphatases s'opposent très vivement à la stimulation de la transcription du gène de la caséine- $\beta$ par le relais de la prolactine (tabl. 2). Il est donc vraisemblable que le relais de la prolactine active une phosphatase nucléaire et que la déphosphorylation des protéines qui en résulte contribue à accélérer la transcription des gènes sensibles à la prolactine.

\section{Conclusion.}

Les quelques données que nous possédons maintenant sur les mécanismes d'action de l'insuline et de la prolactine font apparaître de frappantes analogies. Analogies à double titre puisque les relais présumés des deux hormones partagent un certain nombre de propriétés physicochimiques et puisque les deux relais semblent être des activateurs de phosphatases. II est donc tentant de généraliser et de considérer qu'un nouveau type de mécanisme d'action hormonale a été partiellement élucidé. Cette généralisation est toutefois encore malaisée car trop de questions fondamentales demeurent. La question essentielle à laquelle on aimerait répondre est celle de savoir si les relais de l'insuline et de la prolactine sont une seule et même entité biochimique ou s'ils constituent plutôt une famille de molécules.

Si les relais des deux hormones sont identiques, l'insuline qui possède des récepteurs dans la cellule mammaire devrait avoir des propriétés lactogènes, ce qui de toute évidence n'est pas le cas. II est frappant toutefois de constater que le relais de l'insuline peut stimuler légèrement la transcription du gène de la caséine- $\beta$ et que le relais de la prolactine augmente considérablement l'activité pyruvate déshydrogénase dans un système subcellulaire (Larner, communication personnelle). On peut dès lors imaginer que la prolactine ajoutée à des membranes possédant les récepteurs des deux hormones est beaucoup plus efficace que l'insuline pour provoquer la libération du relais, soit parce que les récepteurs de la prolactine sont plus nombreux ou plus aptes à capter l'hormone, soit parce que le couplage entre le récepteur et le système effecteur membranaire qui pré- 
side à la formation du relais est plus favorable dans le cas du récepteur de la prolactine. Si tel était le cas, la prolactine devrait posséder les propriétés d'une " super-insuline ». II semble que cette proposition ne soit pas acceptable simplement car, s'il est vrai que la prolactine seule ajoutée à des milieux de culture d'explants mammaires de lapine permet une expression significative de la fonction lactogène (Devinoy et al., 1978), ceci n'est plus vrai chez la souris chez laquelle une synergie entre les deux hormones est nécessaire pour permettre une accumulation des RNA messagers des caséines (Bolander et al., 1981). II en est de même pour l'activité mitogène de la prolactine chez la lapine qui ne peut s'exprimer qu'en présence d'insuline (Martel et Houdebine, 1982). Il est vrai toutefois que dans ce dernier cas, l'insuline n'agit qu'à forte concentration et donc via les récepteurs de type IGF, ce qui peut-être vient encore compliquer l'interprétation des expériences réalisées dans les systèmes subcellulaires.

Si les relais des deux hormones sont deux molécules différentes, leur analogie de structure peut être suffisante pour qu'une stimulation croisée puisse se manifester dans les systèmes subcellulaires sans que ceci remette en cause la spécificité d'action des deux hormones in vivo.

Quoi qu'il en soit, la question se pose de savoir si les relais mis en évidence pour la prolactine et pour l'insuline peuvent à eux seuls rendre compte de la multiplicité des actions de ces deux hormones dans leurs cellules cibles respectives. En particulier, les gènes sensibles à l'insuline (Peavy et al., 1978 ; Pry et Porter, 1981 ; Korc et al., 1981) sont-ils activables par le relais de l'insuline et éventuellement par celui de la prolactine. De même, les enzymes activées par la prolactine et qui subissent une déphosphorylation (Hardie et Guy, 1980) sont-elles sensibles aux relais des hormones? En d'autres termes est-il déjà nécessaire d'envisager qu'un groupe de relais plutôt qu'un seul est émis par la membrane après occupation des récepteurs hormonaux? Les expériences décrites par Cheng et al. (1980) et Saltiel et al. (1982) font plutôt pencher dans le sens d'une multitude de relais. Dans la cellule mammaire, bien que les effets mitogène et lactogène de la prolactine soient simultanément inhibés et mimés respectivement par la colchicine (Houdebine et Djiane, 1980) et par les anticorps anti-récepteurs (Djiane et al., 1981), rien ne prouve que les deux signaux aient le même relais et le fait que la prolactine seule est lactogène (Devinoy et al., 1978), mais non mitogène (Martel et Houdebine, 1982) milite plutôt en faveur de la multiplicité des relais.

II est bien évident qu'une réponse réellement significative ne sera donnée à ces questions que lorsque les relais des deux hormones auront été isolés et que leur structure aura été déterminée. Ce projet risque de ne pas aboutir très rapidement en raison de la faible amplitude des stimulations enzymatiques sensibles au relais de l'insuline et en raison de la relative difficulté qu'il y a à évaluer le taux de transcription d'un gène spécifique.

Quel que soit le déroulement futur de ces expériences, il paraît intéressant dès maintenant de tenter d'étendre l'étude des relais hormonaux du type insuline et prolactine à d'autres hormones et à d'autres systèmes, $y$ compris dans les cas où des relais tels que I'AMP cyclique, sont déjà connus. 


\section{Références}

BALOWIN D., TERRIS S., STEINER D. F., 1980. Characterization of insulin like actions of antiinsulin receptor antibodies: effects on insulin binding, insulin degradation and glycogen synthesis in isolated rat hepatocytes. J. biol. Chem., 225, 4028-4034.

BOLANDER F. F., NICHOLAS K. R., VANWYK J. J., TOPPER Y. J., 1981. Insulin is essential for accumulation of casein mRNA in mouse mammary epithelial cells. Proc. nat. Acad. Sci. USA, 78, 5682-5684.

CHENG K., GALASKO G., HUANG L., KELLOG J., LARNER J., 1980. Studies on the insulin mediator II. Separation of two antagonistic biologically active materials from fraction II. Diabetes, 29, 659-661.

COHEN P., 1982. The role of phosphorylation in neural and hormonal control of cellular activity. Nature, 296, 613-619.

DEVINOY E., HOUDEBINE L. M., DELOUIS C., 1978. Role of prolactin and glucocorticoids in the expression of casein genes in rabbit mammary gland organ culture. Quantification of casein mRNA. Biochim. biophys. Acta, 517, 360-366.

DJIANE J., HOUDEBINE L. M., KELLY P. A., 1981. Prolactin-like activity of anti-prolactin receptor antibodies on casein and DNA synthesis in the mammary gland. Proc. nat. Acad. Sci. USA, 78, 7445-7448

FASEL N., PEARSON K., PUETT I., DIEGELMANN H., 1982. The region of mouse mammary tumor virus DNA containing the long terminal repeat includes a long coding sequence and signals for hormonally regulated transcription. EMBO J., 1, 3-7.

HARDIE D. G., GUY P. S., 1980. Reversible phosphorylation and inactivation of Acetyl-CoA carboxylase from lactating rat mammary gland by cyclic AMP dependent protein kinase. Europ. J. Biochem., 110, 167-177.

HOUDEBINE L. M., 1980. Role of prolactin, glucocorticoids and progesterone in the control of casein gene expression. In J. DUMONT, J. NUNEZ, Hormones and cell regulation, vol. 4, 175-196. Elsevier/North Holland biomed. Press.

HOUDEBINE L. M., DJIANE J., 1980. Effects of lysosomotropic agents and of microfilament and microtubule disrupting drugs on the activation of casein gene expression by prolactin in the mammary gland. Mol. cell. Endocr., 17, 1-15.

KAHN C. R., BAIRD K. L., FLIER J. S., JARETT D. B., 1977. Effects of auto-antibodies to the insulin receptor on isolated adipocytes. Studies of insulin binding and insulin action. J. clin. Invest., 60, 1094-1106.

KAHN C. R., BAIRD K. L., JARETT D., FLIER J. S., 1978. Direct demonstration that receptor cross-linking or aggregation is important in insulin action. Proc. nat. Acad. Sci. USA, 75, 4209-4213.

KIECHLE F. L., JARETT L., KOTAGAL N., POPP D. A., 1981. Partial purification from rat adipocyte plasma membranes of a chemical mediator which stimulates the action of insulin on pyruvate dehydrogenase. J. Biochem., 256, 2945-2951.

KORC M., OWERBACH D., QUINTO C., RUTTER W. J., 1981. Pancreatic isletacinar cell interaction : amylase messenger RNA levels are determined by insulin. Science, 213, 351353.

LARNER J., GALASKO G., CHENG K., ROACH A. A., HUANG L., DAGGY P., KELLOG J., 1979. Generation by insulin of a chemical mediator that controls protein phosphorylation and dephosphorylation. Science, 206, 1408-1410.

MARTEL P., HOUDEBINE L. M., 1982. Effect of various drugs affecting cytoskeleton and plasma membranes on the induction of DNA synthesis by insulin, epidermal growth factor and prolactin in mammary explants. Biol. cell., 44, 111-116.

MATHIS D., OUDET P., CHAMBON P., 1980. Structure of transcribing chromatin. Progr. nucleic Acid mol. Biol., 24, 1-54.

MULVIHILL E. R., LEPENNEC J. P., CHAMBON P., 1982. Chicken oviduct progesterone receptor : location of specific regions of high affinity binding in cloned DNA fragments of hormone responsive genes. Cell, 24, 621-632. 
NOLIN J. M., BOGDANOVE E. M., 1980. Effects of oestrogen on prolactin incorporation by lutein and milk secretory cells and on pituitary prolactin secretion in the postpartum rat : correlations with target cell responsivness to prolactin. Biol. Reprod., 22, 3933-3416.

PEAVY D., TAYLOR J. M., JEFFERSON L. S., 1978. Correlation of albumin production rates and albumin mRNA levels in livers of normal diabetic and insulin-treated diabetic rats. Proc. nat. Acad. Sci. USA, 75, 5879-5883.

PRY T. A., PORTER J. N., 1981. Control of fatty acid synthetase mRNA levels in rat liver by insulin, giucagon and dibutyl cyclic AMP. Biochem. biophys. Res. Commun., 100, 10021009.

SALTIEL A. R., SIEGEL M. I., JACOBS S., CUATRECASAS P., 1982. Putative mediators of insulin action : regulation of pyruvate dehydrogenase and adenylate cyclase activities. Proc. nat. Acad. Sci. USA, 79, 3513-3517.

SEALS J. A., JARETT L., 1980. Activation of pyruvate deshydrogenase by direct addition of insulin to an isolated plasma membrane/mitochondria mixture. Evidence for generation of insulin's second messenger in a subcellular system. Proc. nat. Acad. Sci. USA, 77, 77-81.

TEYSSOT B., HOUDEBINE L. M., 1980. Role of prolactin in the transcription of $\beta$-casein and $28 \mathrm{~S}$ ribosomal genes in the rabbit mammary gland. Eur. J. Biochem., 110, 263-272.

TEYSSOT B., HOUDEBINE L. M., 1981. Role of progesterone and glucocorticoids in the transcription of the $\beta$-casein and $28 \mathrm{~S}$ ribosomal genes in the rabbit mammary gland. Eur. J. Biochem., 114, 597-608.

TEYSSOT B., HOUDEBINE L. M., DJIANE J., 1981. Prolactin induces release of a factor from membranes capable of stimulating $\beta$-casein gene transcription in isolated mammary cell nuclei. Proc. nat. Acad. Sci. USA, 78, 6729-6733.

WHITEHOUSE S., COOPER R. H., RANDLE P. J., 1974. Mechanism of activation of pyruvate dehydrogenase by dichloroacetate and other halogenated carboxylic acids. Biochem. $J$., 141, $761-774$. 